gehört integral zu diesem mehrschichtigen Werk. Auch wer „religiös unmusikalisch“ (Max Weber) ist, sollte sich von der Auseinandersetzung mit ihm nicht abschrecken lassen. Es zählt neben Thomas Pikettys neuem Buch, das ebenfalls zum Teil ideengeschichtlich vorgeht („Capital et idéologie“, 2019, dt. 2020), zu den Leittexten gegenwärtiger Kapitalismuskritik.

Funding Open Access funding enabled and organized by Projekt DEAL.

Open Access Dieser Artikel wird unter der Creative Commons Namensnennung 4.0 International Lizenz veröffentlicht, welche die Nutzung, Vervielfältigung, Bearbeitung, Verbreitung und Wiedergabe in jeglichem Medium und Format erlaubt, sofern Sie den/die ursprünglichen Autor(en) und die Quelle ordnungsgemäß nennen, einen Link zur Creative Commons Lizenz beifügen und angeben, ob Änderungen vorgenommen wurden.

Die in diesem Artikel enthaltenen Bilder und sonstiges Drittmaterial unterliegen ebenfalls der genannten Creative Commons Lizenz, sofern sich aus der Abbildungslegende nichts anderes ergibt. Sofern das betreffende Material nicht unter der genannten Creative Commons Lizenz steht und die betreffende Handlung nicht nach gesetzlichen Vorschriften erlaubt ist, ist für die oben aufgeführten Weiterverwendungen des Materials die Einwilligung des jeweiligen Rechteinhabers einzuholen.

Weitere Details zur Lizenz entnehmen Sie bitte der Lizenzinformation auf http://creativecommons.org/ licenses/by/4.0/deed.de.

\title{
Robichaud, Andrew A.: Animal City. The Domestication of America, 352 S., Harvard UP, Cambridge, MA/London 2019.
}

\author{
Andreas Hübner \\ Online publiziert: 22. September 2020 \\ (C) Der/die Autor(en) 2020
}

Den Blick auf die Flut an Arbeiten gerichtet, die sich dem Themenfeld der HumanAnimal Studies widmeten, sprach die Historikerin Harriet Ritvo im Jahr 2007 von einem animal turn in den Geistes- und Sozialwissenschaften. Über ein Jahrzehnt später hält die Konjunktur der Human-Animal Studies an. In ihrem Sog haben sich auch die historischen Tierforschungen maßgeblich konturiert, professionalisiert und institutionalisiert. Zumeist den Prämissen sozial- und kulturgeschichtlicher Ansätze

\footnotetext{
A. Hübner $(\bowtie)$

Leuphana Universität Lüneburg, Lüneburg, Deutschland

E-Mail: andreas.huebner@leuphana.de
} 
verpflichtet, erkunden Historiker_innen heute die Historizität des Tieres und wählen vor allem Zugänge, die Praktiken, Körper und Räume von Mensch-Tier-Beziehungen ins Zentrum der Untersuchungen stellen. In der Regel dominieren, wie die Historikerin Mieke Roscher unlängst feststellte, in der Tierhistoriografie die Studien der Mensch-Tier-Raumbeziehungen, deren Grundlagen wiederum in den Forschungen der historischen Animal Geography zu sehen sind. In diesem Forschungsrahmen ist auch der 2019 erschienene Band „Animal City“ des Bostoner Umwelthistorikers Andrew Robichaud zu verorten.

Tiere, so notiert Robichaud gleich zu Beginn, seien zentral dafür gewesen, wie Amerikaner_innen die Welten des ausgehenden 19. und des beginnenden 20. Jahrhunderts erklärt und verstanden hätten. Die Menschen hätten mit Tieren gedacht und die Transformationsprozesse, die seit den 1820er Jahren die amerikanische Nation prägten, in ihren Verhältnissen zu Tieren reflektiert. Der Wandel, den Industrialisierung, Mechanisierung und Urbanisierung herbeiführten, bedeutete nichts weniger als eine „radikale Umgestaltung des menschlichen und tierischen Lebens in Amerika“ (S. 4, Übers. d. Verf.).

Es ist demnach nur folgerichtig, wenn Robichaud von der Annahme ausgeht, dass die Entwicklungen moderner, urbaner Räume ohne eine Betrachtung der ,tierischen“ Städte des 19. Jahrhunderts nicht nachzuvollziehen seien. Schließlich erfuhren städtisches Tierleben und Tierindustrien, von Behörden für Stadtplanung und -entwicklung initiiert, spätestens seit den 1830er Jahren eine räumliche Rekonfiguration. Über neuartige Vorstellungen von öffentlicher Hygiene und Gesundheitspflege wurde der Weg für die Reform des städtischen Ordnungsgefüges gebahnt und ein Wandel urbaner Mensch-Tier-Beziehungen befördert. Um diesen Wandel zu beschreiben, widmet sich Robichaud einer Auswahl von Themen, die die gängigen Forschungsinteressen der urbanen Tierforschung widerspiegeln: Er folgt den Spuren der Milch- und Fleischindustrie, der Tierrechtsbewegung und der Haustiergeschichte sowie den Spuren der Zoos, der Zoologischen Gärten und anderer Formen der Zurschaustellung von Tierkörpern aus Unterhaltungs- und Vergnügungszwecken. Dabei bedient er sich der Methoden der Sozial- und Umweltgeschichte. Kulturgeschichtliche und -wissenschaftliche Ansätze spielen eine untergeordnete Rolle. Zur Frage der Animal Agency, die zu den spannendsten Fragen der Human-Animal Studies und der historischen Tierforschung zählt, positioniert sich Robichaud nicht, auch wenn er erkennen lässt, dass die Subjekte seiner Untersuchung den städtischen Tieren durchaus eine Agency zugestanden (vgl. S. 189).

Aus konzeptueller Perspektive zu vermerken ist überdies, dass die Studie den Fährten der urbanen Mensch-Tier-Beziehungen vornehmlich in zwei Städten folgt: Mit New York City wählt Robichaud die gewachsene Metropole an der amerikanischen Ostküste und stellt ihr San Francisco, die „Instant City“ an der Pazifikküste, gegenüber. Während San Francisco durch den Goldrausch in Kalifornien nach 1848 rasant zu wachsen begann, konnte New York City bereits um 1850 auf eine über zweihundertjährige Geschichte zurückblicken und mehr als 500.000 Einwohner_innen in den verschiedenen Stadtbezirken verzeichnen. Entsprechend wirkten sich die urbanen Transformationsprozesse in den beiden Städten sehr unterschiedlich aus. In New York City bedeuteten die Prozesse, die in den 1820er und 30er Jahren einsetzten, eine umfassende Rekonfiguration der lokalen Mensch-Tier-Bezie- 
hungen. Zum einen wurden auf Anraten urbaner Reformer in dieser Zeit zentrale Weideflächen und Gemeindegüter in Orte der Naherholung umgewandelt. Die neu geschaffenen Parks und Gärten waren dezidiert durch Zugangsbeschränkungen für beziehungsweise durch die Abwesenheit von Tieren definiert. Zum anderen stellte die aufkommende Lebensmittelreformbewegung die Praktiken der urbanen Milchviehhaltung und des Milchviehbetriebs infrage. In der Folge verschwanden nicht nur die Kühe selbst aus Manhattan, sondern auch die milchverarbeitenden Gewerbe wurden allmählich aus den innerstädtischen Gebieten verdrängt. Begleitet wurden diese Entwicklungen, die im Bereich der Milchindustrie letztendlich zu einer räumlichen Trennung von Konsumenten und Produzenten führten, von den frühen Bemühungen der Tierschutzbewegung und den aufkommenden Diskursen um die öffentliche Gesundheit (S. 46).

In San Francisco hingegen eröffnete die stete Ausweitung des urbanen Raumes alternative Möglichkeiten städteplanerischer Anpassungen und Neuordnungen. Besonders anschaulich zeichnet Robichaud die Entwicklungen für die Schlacht- und Fleischindustrie nach. Ursprünglich im Bereich des heutigen Mission District und Mission Bay beheimatet, wurden die Klein- und Großbetriebe im Laufe des 19. Jahrhunderts kontinuierlich an den urbanen Rand verlagert. So entstand bis 1910 in direkter Nähe zur San Francisco Bay am südöstlichen Rand der Stadt das berüchtigte Butchertown. Für Reformer und Stadtobere ging damit ein lang gehegter Wunsch in Erfüllung; dank ihrer Bemühungen war das Zentrum San Franciscos zu einem viehfreien und in ihren Augen ,sauberen, respektablen und gesunden Ort“ geworden (S. 65). Gleichwohl schufen sie mit Butchertown langfristig, so argumentiert Robichaud, ,eine der Umweltverschmutzung gewidmete Zone“ (S. 124).

Am Beispiel der Schlacht- und Fleischindustrie in San Francisco gelingt es Robichaud, die historischen Verflechtungen von Mensch-Tier-Beziehungen, Umweltverschmutzung und Rassismus im städtischen Raum offenzulegen. Unter anderem legt er dar, wie die Abgeordneten der Bay City per Verordnung in den späten 1880er Jahren erfolglos versuchten, in unmittelbarer Nachbarschaft von Butchertown ein Wohndistrikt zu schaffen, das speziell der chinesischen Bevölkerung der Stadt zugewiesen werden sollte. Zwar scheiterten die Stadtoberen an gerichtlichen Verfügungen - die Richter erkannten in dem Vorhaben eine Verletzung der Bürgerrechte -, ihre Bemühungen, marginalisierte Gruppen in umweltverschmutzten und gesundheitsgefährdenden Gebieten am Rand der Stadt zu relokalisieren, stellten sie aber nicht ein. Mit Ende des Zweiten Weltkrieges sollte um Butchertown ein afroamerikanisches „ghetto“ entstehen, bei dessen Anblick der Autor James Baldwin kommentierte, man finde an diesem Ort jenes San Francisco, ,von dem Amerika vorgebe, es existiere nicht" (S. 125).

Auch in seinen Ausführungen zur Tierrechtsbewegung und Haustiergeschichte kann Robichaud die Transformationen des urbanen Mensch-Tier-Verhältnisses überzeugend nachzeichnen. Die einschlägigen Schriften von Historiker_innen wie Susan Pearson und Jessica Wang erweiternd, diskutiert er, wie auf Bestreben der New Yorker Society for the Prevention of Cruelty to Animals der Einsatz von Arbeitshunden eingeschränkt wurde. Zur Mitte des 19. Jahrhunderts oftmals vor Karren gespannt oder als Antriebskraft auf Laufrädern eingesetzt, verschwanden Hunde bis zur Jahrhundertwende nahezu vollständig aus der urbanen Arbeitswelt. Stattdessen wurden 
die Vierbeiner als Haus- und Schoßhunde nun in ein humanistisches Ideal der Haustierhaltung eingepasst. Die einst vielfältigen Beziehungen zwischen Menschen und Hunden waren fortan auf die Rollenverteilung von Halter und Haustier reduziert (S. 195).

Der Wandel in den Beziehungen zwischen Menschen und Hunden, so Robichaud, ließ eine grundlegende Neuordnung urbaner Räume erkennen: Mit Beginn des 20. Jahrhunderts waren viele Tiere, die vormals die Straßen, Parks, Läden und Fabriken von New York City und San Francisco gesäumt hatten, aus den städtischen Lebenswelten verschwunden. Dementgegen erlebten die vielfältigen Formen der Zurschaustellung von Tieren, ob klassische Wandermenagerien, Zoologische Gärten, Zoos oder Zirkusse, eine Konjunktur. John Berger hatte in seinem einschlägigen Essay „Why Look at Animals?“ einst festgehalten: „Überall verschwinden Tiere. In Zoos bilden sie das lebende Denkmal für ihr eigenes Verschwinden“. Robichaud ergänzt diese Beobachtung nun, indem er bemerkt, dass mit ihrem Verschwinden auch die Gewalt gegenüber Tieren räumlich verschoben und verborgen wurde (S. 255). Den Kern der Transformation der urbanen Mensch-Tier-Beziehungen beschreibt er wie folgt: Um 1920, als die stete Urbanisierung in Amerika einen ersten Höhepunkt erreichte, waren die Städte weitgehend von den ehemals alltäglichen Tierwelten und eklatanten Tierleiden ,gereinigt' worden. Zugleich wurden Tiere nun als häusliche Gefährten konzipiert, in neue Formen der Vergnügung und Freizeitgestaltung eingepasst oder in liminale Lebensräume verdrängt (S. 264).

Mit seiner Studie legt Robichaud für den nordamerikanischen Raum eine breit angelegte Analyse urbaner Mensch-Tier-Beziehungen vor. Für die Nachhaltigkeit seiner Argumentation wäre es dabei sicherlich produktiv gewesen, die Untersuchungen und Diskussionen auch auf die Städte des amerikanischen Südens auszuweiten. Das aufstrebende Atlanta oder New Orleans, die „Crescent City“ am Mississippi, hätten hierfür naheliegende Ausgangspunkte geboten. Nichtsdestotrotz bleibt festzuhalten, dass Robichaud mit „Animal City“ durchaus überzeugen kann: Er unterzieht seine Beobachtungen einer klugen Analyse, fasst pointiert wie konzise zusammen und legt letztlich eine Studie vor, die die Bedeutung städtischer Mensch-Tier-Beziehungen für unser Verständnis von den urbanen Transformationsprozessen des 19. Jahrhunderts deutlich veranschaulicht.

Funding Open Access funding enabled and organized by Projekt DEAL.

Open Access Dieser Artikel wird unter der Creative Commons Namensnennung 4.0 International Lizenz veröffentlicht, welche die Nutzung, Vervielfältigung, Bearbeitung, Verbreitung und Wiedergabe in jeglichem Medium und Format erlaubt, sofern Sie den/die ursprünglichen Autor(en) und die Quelle ordnungsgemäß nennen, einen Link zur Creative Commons Lizenz beifügen und angeben, ob Änderungen vorgenommen wurden.

Die in diesem Artikel enthaltenen Bilder und sonstiges Drittmaterial unterliegen ebenfalls der genannten Creative Commons Lizenz, sofern sich aus der Abbildungslegende nichts anderes ergibt. Sofern das betreffende Material nicht unter der genannten Creative Commons Lizenz steht und die betreffende Handlung nicht nach gesetzlichen Vorschriften erlaubt ist, ist für die oben aufgeführten Weiterverwendungen des Materials die Einwilligung des jeweiligen Rechteinhabers einzuholen.

Weitere Details zur Lizenz entnehmen Sie bitte der Lizenzinformation auf http://creativecommons.org/ licenses/by/4.0/deed.de. 